\title{
Optimal precoder designs for sum rate maximization in MIMO multiuser multicells
}

- Ha Hoang Kha

- Nguyen Dinh Long

- Tuan Do-Hong

Ho Chi Minh city University of Technology, VNU-HCM, Vietnam

(Manuscript Received on July 15, 2015, Manuscript Revised August 30, 2015)

\begin{abstract}
This paper is concerned with the joint linear precoder design problem for the multiuser multiple-input multiple-output (MIMO) heterogeneous networks (HetNets) in which multiple femto base stations (FBSs) coexist with a macro base station (MBS). To tackle the inter-user interference in the macrocell, we exploit the blockdiagonalization scheme and then use the convex optimization to maximize the sum rate of the macrocell. The FBS transmission strategy is to maximize the sum-rate of femtocells subject to the transmitted power
\end{abstract}

constraints per FBS and restrictions on the cross-tier interference to macro-users (MUs). Such a design problem is typically nonconvex, and, thus, challenging to find the FBS precoders. We reformulate the design problem of the FBS precoders as a d.c. (difference of convex functions) programming, and develop an efficient iterative algorithm to obtain the optimal precoders. Numerical simulation results show that the proposed algorithm outperforms the other methods in terms of the total sum-rate of the HetNet.

Keywords: Linear precoders, MIMO interference channels, multicells, HetNets, d.c. programming.

\section{INTRODUCTION}

Heterogeneous networks (HetNets) have recently become a major research topic in wireless communications due to its great potential to improve the coverage and capacity of wireless networks [1], [2]. In Long Term Evolution (LTE) Advanced HetNets, micro, pico, and femto cells with low power base stations and short range of coverage can be placed in different locations to improve the spectral efficiency since these cells operate in the licensed spectrum of the macrocell and provide the high quality of service (QoS) for nearby users [1], [3]-[5]. The research of the present paper focuses on the HetNets in which several femtocells are underlaid with a macrocell [5]. Due to the scarce frequency resource, the femto base stations (FBSs) share the same frequency spectrum with the macro base station (MBS), then the cochannel cells result in crosstier interference from the marocell to femtocells and from femtocells to macrocell [2], [6]. In addition, with a few number of femtocells in the macrocell, there are additional interference between femtocells, namely, intra-tier interference. Thus, a key challenge for successful 
deployment of HetNets is how efficiently to handle intra-tier and cross-tier interference.

The present work studies on the downlink transmission of the multiple-input multiple-output (MIMO) HetNets in which an MBS serves with multiple macro-users (MUs) while multiple FBSs communicate with multiple femto-users (FUs) simultaneously. In such system models, the downlink transmission of the base stations (BSs) is modeled as MIMO broadcast channels (BCs) [7]. As shown in [8], the channel capacity of the MIMO BCs can be achieved by dirty paper coding (DPC). However, the complexity of the DPC scheme makes it prohibitive for practical implementation [7]. To overcome the high implementation complexity of DPC, the block diagonalization (BD) scheme was introduced in [9]. The BD scheme aligns interference into the null space of the interference channel matrices and, thus, each user can deploy interference-free channel. However, the aforementioned BD schemes [9] only address the inter-user interference in a cell. In contrast to the MIMO BCs, in HetNets, the MBS and FBSs coexist and form two-tier wireless networks. Then, the useful signals in a cell can cause interference to other cells. In HetNets, interference comes from not only inter-users in the same tier but also cross-tier. Thus, cross-tier interference is the bottleneck to improve the channel capacity of HetNets [10]. Reference [6] uses interference alignment to mitigate the interference between FUs. However, [6] considered the model in which each base station serves single user.

Different from the MIMO BCs in [9] in which the BS only deal with interference to its associated users, the FBS in the HetNets has to avoid causing interference to MUs. Since the MU has a higher priority to access the spectrum, the FUs are considered as the secondary users in the HetNets. The femto transmission strategies should not cause any adverse effect on the MUs. The goal of the paper is to maximize the sum rate of the network. To this end, we develop the transmission strategies of the MBS and FBS such that interference between MU and multiple FUs can be efficiently mitigated under assumption of the perfect global channel state information (CSI) at all terminals. The CSI exchange between MBS and FBSs can be done via the low-latency backhaul [10]. The signal transmission between the MBS and MUs is modeled as MIMO BCs. Thus, we employ the BD scheme to cancel interMU interference, and, then exploit the convex optimization to find the MBS precoders such that the sum-rate of the macro-cell is maximized. Next, the FBS transmission should be handle both intra-tier interference to FUs and cross-tier interference to MUs. Therefore, the FBS precoders are designed such that the sum-rate of femtocells is maximized while interference from the FBSs to MUs is restricted below the acceptable threshold. Such a design problem appears mathematically intractable since it is hingly nonlinear and nonconvex. Our proposed approach is to reformulate the design problem into a d.c. (difference of convex functions) programming and, then develop a provably convergent iterative algorithm to find the FB precoders. In each iteration, a convex optimization is efficiently solved by interior-point methods [11]. In simulations, we show that the proposed algorithm is converged in less than 20 iterations. In addition, the numerical results indicate that the proposed method offers higher sum-rate than the time-division multiple access (TDMA) scheme [1] and selfish transmission strategy [12].

The remainder of this paper is organized as follows. Section II introduces the HetNet models considered in the paper. The transmission strategies for the MBS and FBS transmitters are presented in Section III. Section IV illustrates the performance of the proposed method. Finally, Section V provides the concluding remarks.

Notations: Boldface upper and lowercase letters denote matrices and vectors, respectively. The transposition and conjugate transposition of matrix $\mathbf{X}$ are respectively represented by $\mathbf{X}^{\mathrm{T}}$ and $\mathbf{X}^{\mathrm{H}} \cdot \mathbf{X}_{(:, \mathrm{i})}$ denotes the i-th column of matrix $\mathbf{X}$ 
while $\mathbf{X}_{(: \mathrm{i}, \mathrm{j})}$ are the matrix consisting of $(j-i+1)$ columns from the i-th column of matrix $\mathbf{X} . \boldsymbol{I}$ and $\boldsymbol{O}$ stand for identity and zero matrices with the appropriate dimensions. trace(:), $\operatorname{rank}(:)$ and $\mathbb{E}(:)$ are the trace, rank and expectation operators, respectively. $\|\mathbf{x}\|_{2}$ is the Euclidean norm while $\|\mathbf{X}\|_{\mathrm{F}}$ is the Frobenius norm. A Gaussian random vector with mean $\overline{\boldsymbol{x}}$ and covariance $\mathbf{R}_{\mathbf{x}}$ is denoted by $\mathbf{x} \sim \mathcal{C N}\left(\overline{\boldsymbol{x}}, \mathbf{R}_{\mathbf{x}}\right)$.

\section{SYSTEM MODEL}

We consider the downlink of two-tier cellular wireless networks in which one MBS and $K$ FBSs coexist to share the common frequency resource as illustrated in Figure 1. The MBS is equipped with $M_{K+1}$ antennas and serves $I_{K+1}$ macrousers (MUs) equipped with $N_{(K+1)_{i}}$ antennas for the i-th user. The $k$-th FBS simultaneously serves $I_{k}$ femto users (FUs) in its coverage range. The $k$-th FBS has $M_{k}$ antennas while the i-th FU in the $k$-th cell with $k \in K=\{1,2, \ldots, \mathrm{K}\}$ is equipped with $N_{k_{i}}$ antennas.
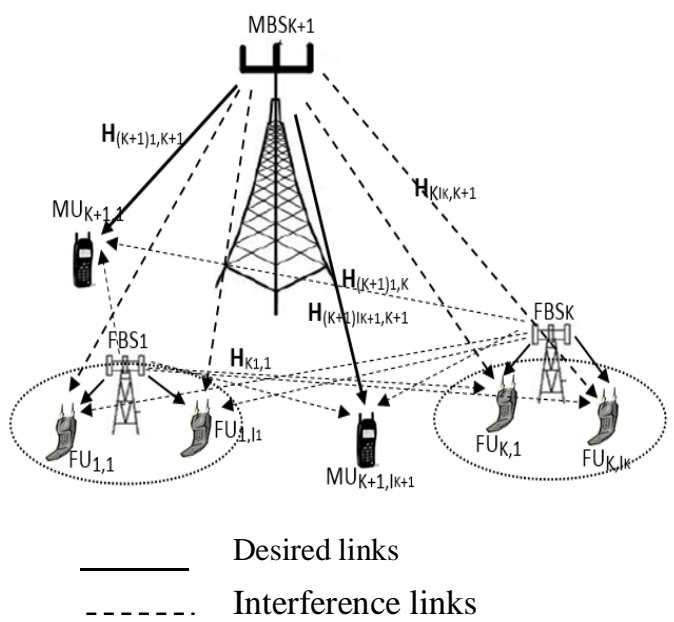

Figure 1. The system model for the downlink in MIMO heterogeneous networks.

Let $S_{k_{i}} \in C^{d_{k_{i}} x_{1}}$ be the $d_{k_{i}}$ independent data streams to be transmitted from the $k$-th BS to the $j$-th user in the $k$-th cell with $j \in\left(1, \ldots, I_{k}\right)$ and $k \in(1, \ldots, K+1)$. The transmitted symbols $s_{k_{i}}$ are assumed to be independent and identically distributed with $\mathbf{E}\left[\boldsymbol{s}_{k_{j}} \boldsymbol{s}_{k_{j}}^{H}\right]=\boldsymbol{I}$. Signal $s_{k_{i}}$ is linearly processed by precoder $V_{k_{j}} \in C^{M_{k} x d_{k_{j}}}$ and, thus, the signal transmitted from the $k$-th BS is

$$
\boldsymbol{x}_{k}=\sum_{j=1}^{I_{k}} \boldsymbol{V}_{k_{j}} \boldsymbol{s}_{k_{j}} .
$$

Accordingly, the transmitted power constraint at the $k$-th $\mathrm{BS}$ is given by

$$
P_{k}=\mathrm{E}\left\{\boldsymbol{x}_{k}^{H} \boldsymbol{x}_{k}\right\}=\sum_{j=1}^{I_{k}} \operatorname{trace}\left(\boldsymbol{V}_{k_{j}} \boldsymbol{V}_{k_{j}}^{H}\right) \leq P_{k}^{\max },
$$

where $P_{k}^{\max }$ is the maximum transmitted power at the $k$-th BS. Assume that the MIMO channels of the links are flat Rayleigh fading and $\boldsymbol{H}_{k_{i}, l} \in C^{N_{k_{i}} x M_{l}}$ is the MIMO channel matrix from the $l$-th BS to the $i$-th user in the $k$-th cell. We also assume that the channels are quasi-static block fading, i.e., they are unchanged during a block transmission and independently changed from block to block [13]. Then, the received signal at the $i$-th user in the $k$-th cell is expressed as

$$
\boldsymbol{y}_{k_{i}}=\sum_{l=1}^{K+1} \boldsymbol{H}_{k_{i}, l} \boldsymbol{x}_{l}+\boldsymbol{n}_{k_{i}}=\sum_{l=1}^{K+1} \sum_{j=1}^{I_{l}} \boldsymbol{H}_{k_{i}, l} \boldsymbol{V}_{l_{j}} \boldsymbol{s}_{l_{j}}+\boldsymbol{n}_{k_{i}},
$$

where $\boldsymbol{n}_{k_{i}} \in C^{N_{k_{i}} x 1}$ is additive white Gaussian noise at the $i$-th user in the $k$-th cell with $n_{k_{i}} \sim C N\left(0, \sigma_{k_{i}}^{2} I_{N_{k_{i}}}\right)$. To clearly analyze the received signals, (3) is rewritten as

$$
\begin{aligned}
\boldsymbol{y}_{k_{i}}= & \boldsymbol{H}_{k_{i}, \mathrm{k}} \boldsymbol{V}_{k_{i}} \boldsymbol{s}_{k_{i}}+\sum_{j=1, j \neq i}^{I_{k}} \boldsymbol{H}_{k_{i}, \mathrm{k}} \boldsymbol{V}_{k_{j}} \boldsymbol{s}_{k_{j}}+ \\
& \sum_{l=1, l \neq k}^{K+1} \sum_{j=1}^{I_{l}} \boldsymbol{H}_{k_{i}, l} \boldsymbol{V}_{l_{j}} \boldsymbol{s}_{l_{j}}+\boldsymbol{n}_{k_{i}},
\end{aligned}
$$

where the first term is the desired signal of the $i$-th user in the $k$-th cell, the second term is intracell interference in the $k$-th cell, the third term is 
the inter-cell interference and the last term is noise at the $i$-th user in the $k$-th cell.

From (4), we can calculate the channel capacity of the $i$-th user in $k$-th cell as follows

$$
C_{k_{i}}=\log _{2}\left|\boldsymbol{I}_{N_{k_{i}}}+\boldsymbol{H}_{k_{i}, k} \boldsymbol{V}_{k_{i}} \boldsymbol{V}_{k_{i}}^{H} \boldsymbol{H}_{k_{i}, k}^{H} \boldsymbol{\Psi}_{k_{i}}^{-1}\right|,
$$

where $\boldsymbol{\Psi}_{k_{i}}=\sigma_{k_{i}}^{2} \boldsymbol{I}_{N_{k_{i}}}+\sum_{(l, j) \neq(k, i)} \boldsymbol{H}_{k_{i}, 1} \boldsymbol{V}_{l_{j}} \boldsymbol{V}_{l_{j}}^{H} \boldsymbol{H}_{k_{i}, l}^{H}$ is the interference-plus-noise covariance matrix. As a result, the total of capacity of the entire system can be expressed as

$$
C_{\text {total }}=\sum_{k=1}^{K+1} \sum_{i=1}^{I_{k}} C_{k_{i}} .
$$

It is obvious from (4) that the received signal suffers from not only intra-tier inference but also cross-tier interference. Such interferences can significantly degrade the data rate or destructively affect to transmission reliability. Our research of interest is to seek the transmission strategies of the MBS and FBSs in order to efficiently mitigate interference.

\section{DESIGN STRATEGIES}

This section will introduce the transmission strategies of the MBS and FBSs such that the sum rate performance of the system is maximized. Since the MBS can exploit the spectrum frequency without awareness of the existence of the FBSs, we first derive the MBS transmission strategy. Then, the FBSs transmission scheme is introduced to handle both intra-tier and cross-tier interference.

\subsection{MBS transmission strategies}

In practice, the FBS operates in the plug and play mode. Thus, the MBS transmission strategy is oblivious the existence of the FBSs and the FBS must guarantee the harmless interference levels to MUs [6]. With the exclusion of cross-tier interference, the received signal of the $i$-th MU in the MBS is given by

$$
\begin{aligned}
& \boldsymbol{y}_{(K+1)_{i}}=\boldsymbol{H}_{(K+1)_{i},(K+1)} \boldsymbol{V}_{(K+1)_{i}} \boldsymbol{s}_{(K+1)_{i}}+ \\
& \sum_{j=1, j \neq i}^{I_{K+1}} \boldsymbol{H}_{(K+1)_{i},(K+1)} \boldsymbol{V}_{(K+1)_{j}} \boldsymbol{s}_{(K+1)_{j}}+\boldsymbol{n}_{(K+1)_{i}} .
\end{aligned}
$$

To remove intra-cell interference at the $i$-th user in the macro cell, we adopt the BD scheme [14]. Form Eq. (7), the conditions for zerointerference are given by

$$
\boldsymbol{H}_{(K+1)_{i},(K+1)} \boldsymbol{V}_{(K+1)_{j}}=0, \forall j \neq i ; i, j=1, \ldots, I_{K+1}
$$

It immediately implies that the channel capacity of the $i$-th user in the macro-cell is given by

$$
\begin{array}{r}
C_{(K+1)_{i}}=\log _{2} \mid \boldsymbol{I}_{N_{(K+1)_{i}}}+\frac{1}{\sigma_{(K+1)_{i}}^{2}} \boldsymbol{H}_{(K+1)_{i},(K+1)} \\
\boldsymbol{V}_{(K+1)_{i}} \boldsymbol{V}_{(K+1)_{i}}^{H} \boldsymbol{H}_{(K+1)_{i},(K+1)}^{H} \mid .
\end{array}
$$

Define $\mathbf{H}_{(K+1)_{j}}$ as the $\left(\sum_{j=1, j \neq i}^{I_{K+1}} N_{(K+1)_{j}}\right) \mathrm{xM}_{K+1}$ channel matrix for all users other than the $j$-th user in the MBS

$$
\begin{aligned}
& \mathbf{H}_{(K+1)_{j}}=\left[\boldsymbol{H}_{(K+1)_{1}}^{T} \ldots \boldsymbol{H}_{(K+1)_{(j-1)}}^{T} \boldsymbol{H}_{(K+1)_{(j+1)}}^{T} \ldots\right. \\
& \left.\ldots \boldsymbol{H}_{(K+1)_{(\mathrm{K}+1)}^{T}}^{T}\right]^{T} \text {. }
\end{aligned}
$$

Condition (8) is equivalent to

$$
\mathbf{H}_{(K+1)_{j}} \boldsymbol{V}_{(K+1)_{j}}=0, \quad j=1, \ldots, I_{K+1} .
$$

In other words, $\boldsymbol{V}_{(K+1)_{j}}$ is the null space of matrix $\mathbf{H}_{(K+1)_{j}}$. Accordingly, the null space condition (11) imposes $M_{K+1}-\sum_{j=1, j \neq i}^{I_{K+1}} N_{(K+1)_{j}}$ $\geq d_{(K+1)_{j}}$. Applying the SVD to $\mathbf{H}_{(K+1)_{j}}$ yields

$$
\mathbf{H}_{(K+1)_{j}}=U_{(K+1)_{j}} \sum_{(K+1)_{j}} V_{(K+1)_{j}}^{H},
$$

where $U_{(K+1)_{j}}$ and $V_{(K+1)_{j}}$ are the left and right singular matrices of $\mathbf{H}_{(K+1)_{j}}$. The diagonal matrix $\sum_{(K+1)_{j}}$ contains the decreasing ordered 
singular values on its diagonal. To satisfy (11), we chose

$\boldsymbol{V}_{(K+1)_{j}}=\boldsymbol{V}_{(K+1)_{j}}\left(:, M_{K+1}-d_{(K+1)_{j}}+1: M_{K+1}\right) \boldsymbol{T}_{(K+1)}$

where $\boldsymbol{T}_{(K+1)_{j}} \in C^{d_{(K+1)_{j}} \mathrm{x} d_{(K+1)_{j}}}$ can be an arbitrary matrix subject to the power constraint at the MBS. From (2), the transmitted power constraint at the MBS is rewritten as

$$
\begin{aligned}
P_{K+1}= & \sum_{i=1}^{I_{K+1}} \operatorname{trace}\left(\boldsymbol{V}_{(K+1)_{i}} \boldsymbol{V}_{(K+1)_{i}}^{H}\right)= \\
& \sum_{i=1}^{I_{K+1}} \operatorname{trace}\left(\boldsymbol{T}_{(K+1)_{i}} \boldsymbol{T}_{(K+1)_{i}}^{H}\right) \leq P_{K+1}^{\max } .
\end{aligned}
$$

Then, from (9) the sum rate at the MBS can be calculated as

$$
\begin{array}{r}
C_{K+1}=\sum_{i=1}^{I_{K+1}} \log _{2} \mid \boldsymbol{I}_{N_{(K+1)_{i}}}+\frac{1}{\sigma_{(K+1)_{i}}^{2}} \boldsymbol{H}_{(K+1)_{i}} \\
\boldsymbol{T}_{(K+1)_{i}} \boldsymbol{T}_{(K+1)_{i}}^{H} \boldsymbol{H}_{(K+1)_{i}}^{H} \mid,
\end{array}
$$

where we define $\boldsymbol{H}_{(K+1)_{i}}=\boldsymbol{H}_{(K+1)_{i},(K+1)} \boldsymbol{V}_{(K+1)_{i}}$ $\left(:, M_{K+1}-d_{(K+1)_{j}}+1: M_{K+1}\right)$ for simplicity.

The design problem of interest is to find the precoders at the MBS to maximize the total sum rate. Thus, the optimal design of precoders can be mathematically posed as

$$
\begin{aligned}
\max _{\boldsymbol{Q}_{(K+1)_{i}}} \sum_{i=1}^{I_{K+1}} \log _{2}\left|\boldsymbol{I}_{N_{(K+1)_{i}}}+\frac{1}{\sigma_{(K+1)_{i}}^{2}} \boldsymbol{H}_{(K+1)_{i}} \boldsymbol{Q}_{(K+1)_{i}} \boldsymbol{H}_{(K+1)_{i}}^{H}\right| \\
\text { s.t. } \quad \sum_{i=1}^{I_{K+1}} \operatorname{trace}\left(\boldsymbol{Q}_{(K+1)_{i}}\right) \leq P_{K+1}^{\max }
\end{aligned}
$$

where $\boldsymbol{Q}_{(K+1)_{i}}=\boldsymbol{T}_{(K+1)_{i}} \boldsymbol{T}_{(K+1)_{i}}^{H}$. It is clear that the objective function and constraints of (16) are convex optimization. It is well known that in such a convex optimization problem, a local optimum is also a global optimum. Thus, problem (16) can be efficiently solved by standard optimization software packages, e.g., CVX [15]. After obtaining the optimal solution $\boldsymbol{Q}_{(K+1)_{i}}$ to problem (16), we calculate the singular value decomposition $\boldsymbol{Q}_{(K+1)_{i}}=\boldsymbol{U}_{(K+1)_{i}} \sum_{(K+1)_{i}} \boldsymbol{V}_{(K+1)_{i}}^{H}$ and obtain the optimal $\boldsymbol{T}_{(K+1)_{i}}$ by

$$
\boldsymbol{T}_{(K+1)_{i}}=\boldsymbol{U}_{(K+1)_{i}} \sum_{(K+1)_{i}}^{1 / 2} .
$$

We summarize the design steps of the precoders at MBS in Algorithm 1.

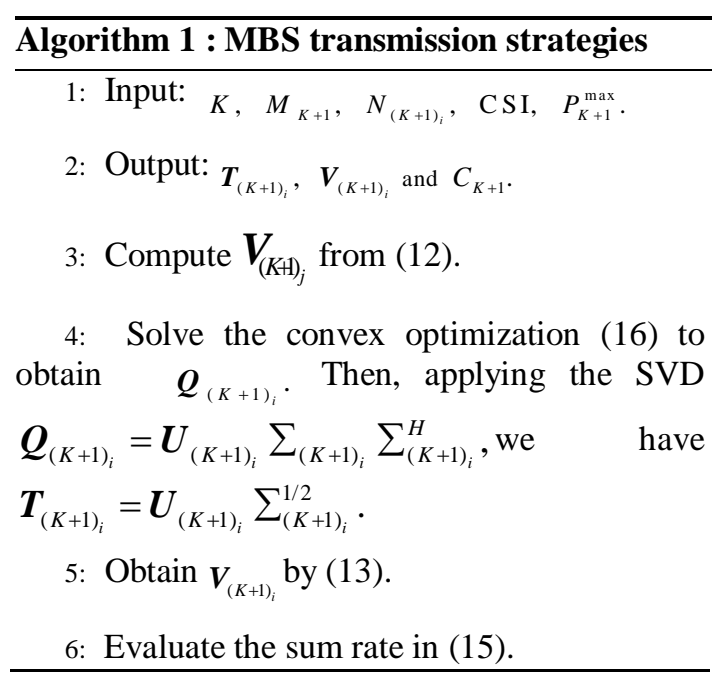

\subsection{FBS transmission strategies}

Note that the FBSs can operate in the same frequency with the MBS if they do not cause harmful interference to MUs. In addition, the intra-tier interference should be mitigated to enhance the sum rate of the femtocells. This means that the FBSs should be deal with both cross-tier and intra-tier interference. The received signal of the $i$-th user in the $k$-th cell, defined by (4), yields the channel capacity of the $i$-th user in $k$-th femtocell given by

$$
\begin{gathered}
C_{k_{i}}=\log _{2}\left|\boldsymbol{I}_{N_{k_{i}}}+\boldsymbol{H}_{k_{i}, k} \boldsymbol{V}_{k_{i}} \boldsymbol{V}_{k_{i}}^{H} \boldsymbol{H}_{k_{i}, k}^{H} \Psi_{k_{i}}^{-1}\right|, \quad \text { (18) } \\
\text { where } \boldsymbol{\Psi}_{k_{i}}=\sigma_{k_{i}}^{2} \boldsymbol{I}_{N_{k_{i}}}+\sum_{(l, j) \neq(k, i)} \boldsymbol{H}_{k_{i}, 1} \boldsymbol{V}_{l_{j}} \boldsymbol{V}_{l_{j}}^{H} \boldsymbol{H}_{k_{i}, l}^{H} .
\end{gathered}
$$

The sum-rate of the femtocells is

$$
R\left(\left\{\boldsymbol{Q}_{k_{i}}\right\}\right)=\sum_{k=1}^{K} \sum_{i=1}^{I_{k}} \log _{2}\left|\boldsymbol{I}_{N_{k_{i}}}+\boldsymbol{H}_{k_{i}, k} \boldsymbol{Q}_{k_{i}} \boldsymbol{H}_{k_{i}, k}^{H} \boldsymbol{\Psi}_{k_{i}}^{-1}\right| .
$$


It is highly desired that the femtocell deployment still guarantees the quality of service of the MUs. The inter-cell interference from $K$ femtocells caused to the $i$-th user in the MBS must by constrained by an acceptable threshold $\eta_{(K+1)_{i}}$

$$
\tilde{\boldsymbol{I}}_{(K+1)_{i}}=\sum_{k=1}^{K} \sum_{j=1}^{I_{k}} \operatorname{trace}\left(\boldsymbol{H}_{(K+1)_{i}, k} \boldsymbol{Q}_{k_{j}} \boldsymbol{H}_{(K+1)_{i}, k}^{H}\right) \leq \eta_{(K+1)_{i}}
$$

where we define

$\boldsymbol{Q}_{k_{j}}=\boldsymbol{V}_{k_{j}} \boldsymbol{V}_{k_{j}}^{H_{j}}$ with $k=1, \ldots K$ and $j=1, \ldots I_{k}$

For simplicity of notation, we define $\boldsymbol{Q}=\left\{\boldsymbol{Q}_{k_{j}}\right\}_{k=1, \ldots, K \text { and } j=1, \ldots, I_{k}}$. To investigate on the efficiency of the precoder design, we consider the scenario in which perfect CSI is exchanged by the BSs via the backhaul links [10] and global CSI is perfectly known at all BSs. The problem of interest is to find the precoders at FBSs to maximize the total sum rate of $K$ femtocells. Thus, the optimal design of precoders can be written as

$$
\begin{gathered}
\max _{\boldsymbol{Q}} R(\boldsymbol{Q}) \\
\text { s.t. } \sum_{i=1}^{I_{k}} \operatorname{trace}\left(\boldsymbol{Q}_{k_{i}}\right) \leq P_{k}^{\max } \quad k=1, \ldots, K \\
\sum_{k=1}^{K} \sum_{j=1}^{I_{k}} \operatorname{trace}\left(\boldsymbol{H}_{(K+1)_{i}, k} \boldsymbol{Q}_{k_{j}} \boldsymbol{H}_{(K+1)_{i}, k}^{H}\right) \\
\leq \eta_{(K+1)_{i}}, \quad i=1, \ldots, I_{K+1}
\end{gathered}
$$

where constraint (22b) is imposed on the transmitted power per FBS while constraint (22c) guarantees that the interference power at the $i$-th MU receiver is less than an allowable threshold $\eta_{(K+1)_{i}} \cdot$

It is obvious that the constraints of (22) are convex while the objective function is nonconcave. Thus, problem (22) is nonconvex which renders the mathematical challenges to find the optimal solutions of (22). Our approach is to recast problem (22) into a d.c. optimization and, then, develop an iterative d.c. programming for finding the precoders. To this end, we rewrite (22) as

$$
\min _{\boldsymbol{Q}} \sum_{k=1}^{K} \sum_{i=1}^{I_{k}}\left[\log _{2}\left|\Psi_{k_{i}}\right|-\log _{2}\left|\Psi_{k_{i}}+\boldsymbol{H}_{k_{i}, k} \boldsymbol{Q}_{k_{i}} \boldsymbol{H}_{k_{i}, k}^{H}\right|\right]
$$

$$
\begin{gathered}
\text { s.t. } \quad \sum_{i=1}^{I_{k}} \operatorname{trace}\left(\boldsymbol{Q}_{k_{i}}\right) \leq P_{k}^{\max } \quad k=1, \ldots, K \\
\sum_{k=1}^{K} \sum_{j=1}^{I_{k}} \operatorname{trace}\left(\boldsymbol{H}_{(K+1)_{i}, k} \boldsymbol{Q}_{k_{j}} \boldsymbol{H}_{(K+1)_{i}, k}^{H}\right) \\
\leq \eta_{(K+1)_{i}}, \quad i=1, \ldots, I_{K+1}
\end{gathered}
$$

where $\boldsymbol{\Psi}_{k_{i}}=\sigma_{k_{i}}^{2} \boldsymbol{I}_{N_{k_{i}}}+\sum_{(l, j) \neq(k, i)} \boldsymbol{H}_{k_{i},} \boldsymbol{Q}_{l_{j}} \boldsymbol{H}_{k_{i}, l}^{H}$. This minimization problem is still nonconvex because $\log _{2}\left|\Psi_{k_{i}}\right|$ concave. Nevertheless, it can be solved by applied the local d.c. programming [16], [17]. Since $\log _{2}\left|\Psi_{k_{i}}\right|$ is concave, at the $\kappa$-thiteration, one can has

$$
\begin{aligned}
\log _{2}\left|\Psi_{k_{i}}\right| \leq \log _{2}\left|\Psi_{k_{i}}^{(\kappa)}\right|+ & \\
& \sum_{(l, j) \neq(k, i)} \operatorname{trace}\left[\boldsymbol{H}_{k_{i}, l}^{H}\left(\boldsymbol{\Psi}_{k_{i}}^{(\kappa)}\right)^{-1} \boldsymbol{H}_{k_{i}, l}\left(\boldsymbol{Q}_{l_{j}}-\boldsymbol{Q}_{l_{j}}^{(\kappa)}\right)\right]
\end{aligned}
$$

Replacing (24) into (23) yields the following optimization problem

$$
\begin{aligned}
\min _{\boldsymbol{Q}} & \sum_{k=1}^{K} \sum_{i=1}^{I_{k}}\left[\log _{2}\left|\Psi_{k_{i}}^{(\kappa)}\right|+\right. \\
& \sum_{(l, j) \neq(k, i)} \operatorname{trace}\left[\boldsymbol{H}_{k_{i}, l}^{H}\left(\boldsymbol{\Psi}_{k_{i}}^{(\kappa)}\right)^{-1} \boldsymbol{H}_{k_{i}, l}\left(\boldsymbol{Q}_{l_{j}}-\boldsymbol{Q}_{l_{j}}^{(\kappa)}\right)\right] \\
& \left.-\log _{2}\left|\Psi_{k_{i}}+\boldsymbol{H}_{k_{i}, k} \boldsymbol{Q}_{k_{i}} \boldsymbol{H}_{k_{i}, k}^{H}\right|\right]
\end{aligned}
$$

s.t. $\sum_{i=1}^{I_{k}} \operatorname{trace}\left(\boldsymbol{Q}_{k_{i}}\right) \leq P_{k}^{\max } \quad k=1, \ldots, K$

$$
\begin{array}{r}
\sum_{k=1}^{K} \sum_{j=1}^{I_{k}} \operatorname{trace}\left(\boldsymbol{H}_{(K+1)_{i}, k} \boldsymbol{Q}_{k_{j}} \boldsymbol{H}_{(K+1)_{i}, k}^{H}\right) \\
\leq \eta_{(K+1)_{i}}, \quad i=1, \ldots, I_{K+1}
\end{array}
$$

which is a convex optimization and, thus, it can be efficiently solved. As a result, the iterative 
procedure to solve problem (23) is summarized in Algorithm 2 where $\epsilon$ is an acceptable accuracy. Note that problem (23) is of d.c. programming and thus, it can be proved that the convergence of Algorithm 2 is guaranteed [16], [17].

\section{Algorithm 2 : Interative algorithm for sum} rate maximization of FBSs

1: Initialization: Set $\kappa=0$, choose $\left\{\boldsymbol{Q}_{k_{i}}^{(0)}\right\}$ , and calculate $R\left(\left\{\boldsymbol{Q}_{k_{i}}^{(0)}\right\}\right)$.

2: $\kappa$-th iteration: Solve the convex optimization problem (25) to obtain the solution $\boldsymbol{Q}_{k_{i}}^{*} \quad$ and $\quad$ set $\quad \kappa=\kappa+1, \quad \boldsymbol{Q}_{k_{i}}^{(\kappa)}=\boldsymbol{Q}_{k_{i}}^{*}$ and calculate $R\left(\left\{\boldsymbol{Q}_{k_{i}}^{(\kappa)}\right\}\right)$.

$$
\begin{gathered}
\text { 3: } \quad \text { Ending } \quad \text { iteration: } \\
\left|\left[R\left(\left\{\boldsymbol{Q}_{k_{i}}^{(\kappa)}\right\}\right)-R\left(\left\{\boldsymbol{Q}_{k_{i}}^{(\kappa-1)}\right\}\right)\right] /\left[R\left(\left\{\boldsymbol{Q}_{k_{i}}^{(\kappa)}\right\}\right)\right]\right| \leq
\end{gathered}
$$

then stop; else go to step 2.

\section{ILLUSTRATIVE RESULTS}

In this section, we evaluate the performance of the proposed method by numerical simulations. We consider the HetNet with $1 \mathrm{MBS}$ and $K=2$ FBSs. Each MBS or FBS is equipped with $M_{k}=$ $M=2$ antennas. Each base station serves $I_{k}=I=$ 2 users, each equipped with $N_{k}=N=2$ antennas. Each base station transmits $\mathrm{d}_{k_{i}}=d=1$ data stream to its intended user. The MIMO Rayleigh channels are randomly generated with zero mean and unit variance entries. All noise variances are normalized $\sigma_{k_{i}}^{2}=\sigma^{2}=1, k=1, \ldots, K+1$ and $i$ $=1, \ldots, I_{k}$. We assume that all FBSs have the same maximum allowable transmitted power $P_{k}^{\text {max }}=P_{\text {max }}$ while the MBS has $P_{K+1}^{\max }=2 P_{\mathrm{max}}$. We investigate the sum rate of the system for $P_{\mathrm{m} \mathrm{ax}}$ from 0 to $30 \mathrm{~dB}$. We set the acceptable interference threshold at all MUs $\eta_{(K+1) i}=\eta$.
Firstly, we study on the convergence characteristic of the proposed Algorithm 2. We set $\eta=0.1$ and $\epsilon=10^{-9}$. Figure 2 illustrates the evolution of the objective function (22) over iterations. It can observed that the objective function is monotonically increased over iterations and it quickly converges in less than 20 iterations. In addition, as the maximum allowable transmitted power increases, the sum rate of the FBSs also increases.

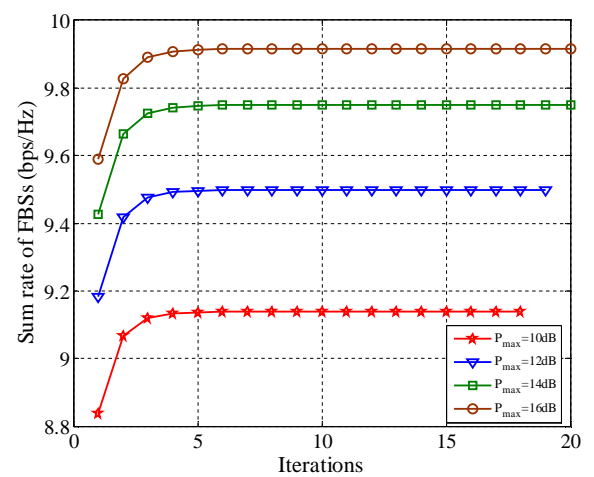

Figure 2. Convergence characteristic of the proposed algorithm.

Next, we investigate the sum rate performance loss of the macrocell for different interference powers caused by FBSs. As can seen from Fig. 3 that for a small fixed value of $\eta=0.1$ the reduction in the sum-rate of the macrocell when there is the presence of FBSs is negligible. When we increase the allowable interference threshold $\eta=1$, the sum-rate performance loss of the marocell increases. When the allowable interference threshold varies with respect to the transmitted power at base stations, $\eta=0.1 P_{\max }$ or $\eta=0.5 P_{\max }$, the sum-rate of the macrocell does not increase when the transmitted power is large enough due to an increasing interference power at MUs. 


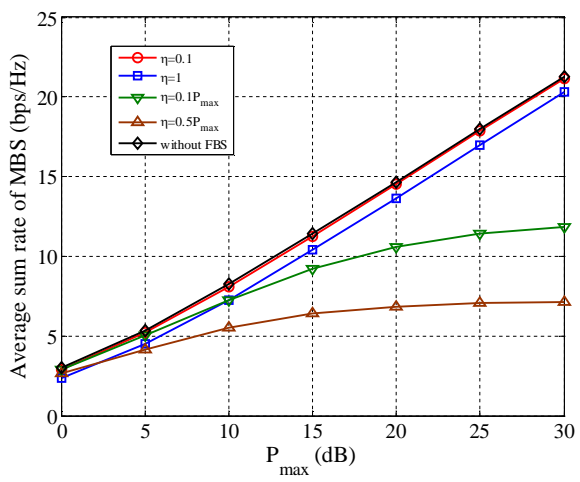

Figure 3. The sum rate loss of the macrocell for difference interference threshold.

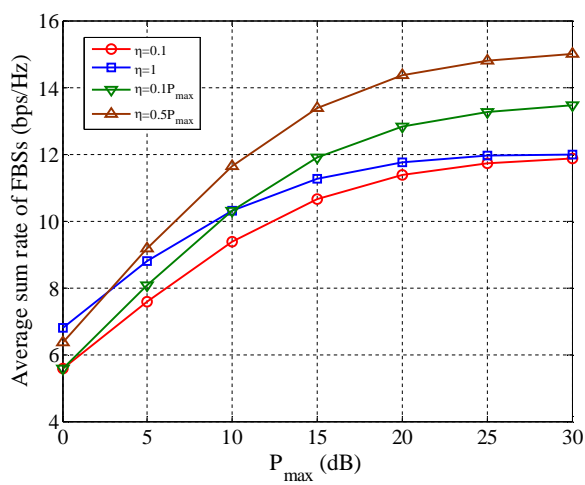

Figure 4. The sum rate of the femtocells for difference interference threshold.

On the other hand, the sum rate of femtocells is given in Fig. 4. In contrast to the macrocell, the average sum-rate of femtocells increases when the interference constraints at the MUs are more relaxed, as shown in Fig. 4. It is observed from Figs. 3 and 4, the average sum rate of the macrocell is higher than that of the femtocells. The reasons are that the MBS has higher transmitted power than the FBSs and the transmission strategies of FBSs must guarantee the harmless interference to MUs.

Now, we compare the total sum-rate performance of our proposed method with that of time division multiple access (TDMA) in [1] and selfish approach in [12]. In TDMA, each base station transmits the signals in different time slots so that there is no inter-cell interference [1]. In selfish approach, each base station only cares about its signals in its own cell and does not care about interference to users in other cells [12]. As observed from Fig. 5, our proposed method outperforms the other methods for all interested region $P_{\max }$.

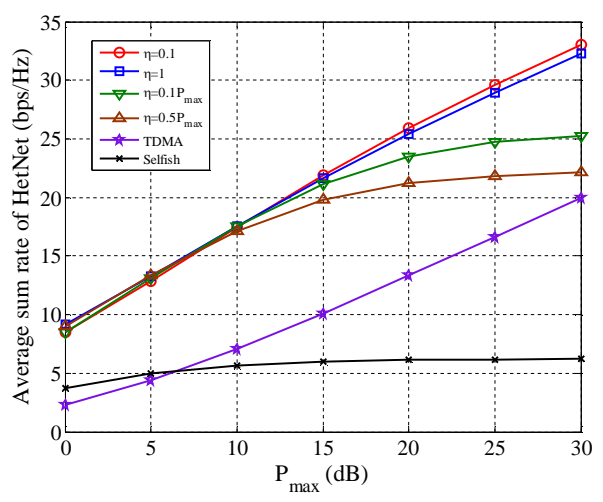

Figure 5. The sum-rate of the HetNet.

Especially, when $P_{\max }$ is large, the sum-rate performance gap between our method and the selfish approach is significant. This is because the inter-cell interference is dominant for large $P_{\max }$ while selfish approach is not aware to inter-cell interference. On the other hand, the TDMA approach can handle inter-cell interference but each cell can use only a part of time for transmission. By choosing an appropriate interference threshold $\eta$, our method can provide an improved sum-rate since it can efficiently handle both inter and intra-cell interference.

\section{CONCLUSION}

This paper has presented the transmission strategies for the downlink of multicell multiuser MIMO HetNets. The block diagonalization scheme is used for the macrocell. The precoders at the FBSs are designed to maximize the total sum-rate while keeping interference to the MUs below the acceptable threshold. The convex optimization is exploited to find the MBS precoders while the d.c. programming is used to find the precoders at the FBSs. The simulation 
results show the effectiveness of the proposed method as compared with the typical TDMA scheme and selfish transmission strategy in terms of the sum rate.

\section{ACKNOWLEDGEMENT}

This research is funded by Vietnam National Foundation for Science and Technology Development (NAFOSTED) under grant number 102.04-2013.46.

\section{Thiết kế bộ tiền mã hóa tối ưu để cực đại tổng tốc độ bit trong hệ thống MIMO nhiều cell nhiều người sử dụng}

- Hà Hoàng Kha

- Nguyễn Đình Long

- Đỗ Hồng Tuấn

Trường Đại học Bách Khoa, ĐHQG-HCM, Việt Nam

\section{TÓM TÁT}

Bài báo nghiên cứu về vấn đề thiết kế các bộ tiền mã hóa tuyến tính cho hệ thống mạng không đồng nhất bao gồm nhiều người sử dụng có nhiều antenna phát và nhiều antenna thu. Mô hình mạng không đồng nhất bao gồm nhiều trạm phát femto hoạt động đồng thời trong vùng phủ sóng của một trạm phát macro. Để giải quyết vấn đề can nhiễu giữa các người sử dụng trong macrocell, chúng tôi sử dụng kỹ thuật khối chéo hóa và kỹ thuật tối ưu lồi để cực đại hóa tổng tốc độ bit của người sử dụng trong macrocell. Kỹ thuật truyền của các trạm femto được thiết kế để tối đa hóa tổng dung lượng của người sử dụng trong femtocell với ràng buộc về công suất phát và mức can nhiễu gây ra cho người sử dụng trong macrocell. Vấn đề thiết kế này tổng quát là bài toán tối ưu không lồi, và việc tìm lời giải tối ưu là một thách thức. Giải pháp của chúng tôi là biến đổi vấn đề thiết kế các bộ tiền mã hóa thành bài toán tối ưu hiệu của hai hàm lồi, và chúng tôi phát triển một giải thuật lặp hiệu quả để tìm các bộ mã hóa tối ưu. Các mô phỏng số đã chỉ ra rằng giải pháp đề xuất cung cấp tổng tốc độ bit cao hơn các phương pháp khác cho mạng không đồng nhất.

Từ khóa: Tiền mã hóa tuyến tính, kênh can nhiễu MIMO, nhiều cell, mạng không đồng nhất, tối ưu D.C.

\section{REFERENCES}

[1]. W. Shin, N. Lee, W. Noh, H.-H. Choi, B. Clerckx, C. Shin, and K. Jang, Hierarchical interference alignment for heterogeneous networks with multiple antennas, IEEE Int. 
Conf. Commun. Workshops (ICC), pp. 1-6, (June 2011).

[2]. K. Shashika Manosha, M. Codreanu, N. Rajatheva, and M. Latva-aho, Powerthroughput tradeoff in MIMO heterogeneous networks, IEEE Trans. Wireless Commun., vol. 13, pp. 4309-4322, (August 2014).

[3]. L. Ho, I. Ashraf, and H. Claussen, Evolving femtocell coverage optimization algorithms using genetic programming, in IEEE 20th Int. Symp. Personal, Indoor and Mobile Radio Communications, pp. 2132-2136, (September 2009).

[4]. H. Du, T. Ratnarajah, M. Sellathurai, and C. Papadias, Reweighted nuclear norm approach for interference alignment, IEEE Trans. Commun., vol. 61, pp. 3754-3765, (September 2013).

[5]. X. Kang, Y.-C. Liang, and H. K. Garg, Distributed power control for spectrumsharing femtocell networks using stackelberg game, in IEEE Int.l Conf. Commun. (ICC), pp. 1-5, (June 2011).

[6]. M. Rihan, M. Elsabrouty, O. Muta, and H. Fumkawa, Iterative interference alignment in macrocell-femtocell networks: A cognitive radio approach, in Int. Symp. Wireless Commum. Systems (ISWCS), pp. 654-658, (August 2014).

[7]. S. Shim, J. S. Kwak, R. Heath, and J. Andrews, Block diagonalization for multiuser mimo with other-cell interference, IEEE Trans. Wireless Commun., vol. 7, pp. 26712681, (July 2008).

[8]. G. Caire and S. Shamai, On the achievable throughput of a multiantenna gaussian broadcast channel, IEEE Trans. Inform. Theory,, vol. 49, pp. 1691-1706, (July 2003).
[9]. Z. Shen, R. Chen, J. Andrews, R. Heath, and B. Evans, Sum capacity of multiuser MIMO broadcast channels with block diagonalization, IEEE Trans. Wireless Commun., vol. 6, pp. 2040-2045, (June 2007).

[10].Z. Xu, C. Yang, G. Li, Y. Liu, and S. Xu, Energy-efficient comp precoding in heterogeneous networks, IEEE Trans. Signal Process., vol. 62, pp. 1005-1017, (February 2014).

[11].S. Boyd and L. Vandenberghe, Convex Optimization. New York, NY, USA: Cambridge University Press, (2004).

[12].M. Razaviyayn, M. Sanjabi, and Z.-Q. Luo, Linear transceiver design for interference alignment: Complexity and computation, IEEE Trans. Information Theory, vol. 58, pp. 2896-2910, (May 2012).

[13].D. Tse and P. Viswanath, Fundamentals of Wireless Communication. New York, NY, USA: Cambridge University Press, (2005).

[14].Q. Spencer, A. Swindlehurst, and M. Haardt, Zero-forcing methods for downlink spatial multiplexing in multiuser MIMO channels, IEEE Trans. Signal Process., vol. 52, pp. 461-471, (February 2004).

[15].M. Grant and S. Boyd, CVX: Matlab software for disciplined convex programming, version 2.1 . http://cvxr.com/cvx, (March 2014).

[16].P. Apkarian and H. D. Tuan, Robust control via concave minimization local and global algorithms, IEEE Trans. Automatic Control, vol. 45, pp. 299-305, (February 2000).

[17].H. Kha, H. Tuan, and H. Nguyen, Fast global optimal power allocation in wireless networks by local d.c. programming, IEEE Trans. Wireless Commun., vol. 11, pp. 510515, (February 2012). 